\title{
New Generation of Magnetic Relaxation Sensors Based on the Melt-Spun FeCoBCu Alloys
}

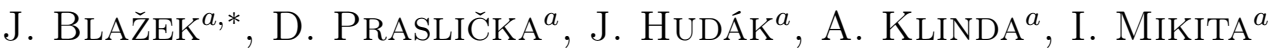 \\ AND J. MARCIN ${ }^{b}$ \\ ${ }^{a}$ Technical University of Košice, Faculty of Aeronautic, Rampová 7, Košice, Slovakia \\ ${ }^{b}$ Institute of Experimental Physics, Slovak Academy of Sciences, Watsonova 47, Košice, Slovakia
}

\begin{abstract}
We report on the development of new generation of magnetic relaxation sensors based on soft magnetic melt-spun ribbons with the composition $\mathrm{Fe}_{63} \mathrm{Co}_{21} \mathrm{~B}_{15} \mathrm{Cu}$. The as-quenched amorphous alloy was subjected to thermomagnetic heat treatment in order to obtain sensor cores with optimized magnetic properties. The relaxation characteristics obtained for these cores by employing upgraded sensor electronics were compared to those based on the model simulations using Matlab-Simulink environment. The operational field range of new developed sensor core reached $300 \mu \mathrm{T}$ within the frequency band $0-500 \mathrm{~Hz}$, i.e. it is markedly extended as compared to the currently used cores based on the commercial VAC 25X material while keeping the similar sensitivity.
\end{abstract}

PACS numbers: $85.90 .+\mathrm{h}$

\section{Introduction}

Relaxation fluxgate sensors are known for their favorable characteristics like simplicity, stability, extraordinary noise resistivity, high dynamics and the ability of a direct conversion to digital signal. Relax-type magnetometer is a pulse energy converter with saturated core, at which the primary quantity proportional to the external magnetic field is the relaxation current, which is independent of the load characteristics. The output relaxation current can be converted into a time interval or voltage in dependence of the characteristics of the load.

The principle of the operation and theoretical analysis of the original relaxation flux gate magnetic sensors can be found in [1,2]. These sensors are excited with short current pulses followed by a relaxation of the accumulated energy into the load represented, as a rule, by a pre-set relaxation diode. The diode determines the sensor relaxation in such a way that the voltage on the sensor winding remains constant while current is reducing linearly. The slope of the current reduction, however, differs by the orders of magnitude when the magnetic core is in saturation state or when the magnetic core is in the state that corresponds to the falling of the field between the saturation levels. The time of relaxation, under such conditions is directly proportional to the amount of the accumulated magnetic energy in the sensor core, whereas it is the very amount of the energy which is determined by the flux density of the measured external magnetic field. The sensor is operating as a direct pulse-width modula-

* corresponding author; e-mail: josef.blazek@tuke.sk tor. There exist, however, several other ways of obtaining and evaluating the measured quantity, e.g. by integration or low frequency filtering during the relaxation current. Thereby a transducer with analogue voltage output is obtained by this way.

The older versions of relaxation fluxgate sensor systems have been used in variety of the industrial and environmental applications as well as in the laboratory magnetometers. To the most successful applications belong the indicators of ferromagnetic bodies developed by the EDIS Company, operating under the most demanding operational conditions at open-cast mines in the Czech Republic.

\section{Material for the sensor core}

A ribbon of the $\mathrm{Fe}_{63} \mathrm{Co}_{21} \mathrm{~B}_{15} \mathrm{Cu}$ amorphous alloy with an average cross-section $6 \mathrm{~mm} \times 25 \mu \mathrm{m}$ was prepared by planar flow casting method at the Institute of Physics, Slovak Academy of Sciences, Bratislava. In order to prepare the samples with preferred direction of induced anisotropy, the pieces of as-quenched ribbon (6 cm long) were isothermally annealed under a high vacuum for $1 \mathrm{~h}$ at $310^{\circ} \mathrm{C}$ slightly below the crystallization temperature in the presence of transverse (TF) or longitudinal (LF) magnetic field. In the case of TF-annealed samples, the furnace was placed inside the commercial permanent magnet system (Magnetic Solutions LTD) producing a magnetic field of $640 \mathrm{kA} / \mathrm{m}$ directed in the plane of the ribbon and perpendicular to its length. In the LF-annealed samples, the furnace was inserted into the water-cooled solenoidal coil that provided a magnetic field of $20 \mathrm{kA} / \mathrm{m}$ oriented along the ribbon length. After such annealing, 
the specimens were slowly cooled to room temperature in a presence of the magnetic field. A typical cooling rate was $3 \mathrm{~K} / \mathrm{min}$. The reference samples were annealed and cooled under the same conditions in a zero magnetic field $(\mathrm{ZF})$. The effects of the magnetic annealing on the hysteresis loops were investigated by using a Forster type hysteresis loop tracer. The focus was given on obtaining cores with optimized BH characteristics such as high value of saturation magnetic flux density and low coercivity and good fluxgate effect.

\section{Results and discussions}

The effect of field annealing on the hysteresis loops of the heat treated $\mathrm{Fe}_{63} \mathrm{Co}_{21} \mathrm{~B}_{15} \mathrm{Cu}$ alloy is demonstrated in Fig. 1. Sheared loop with good field linearity is achieved

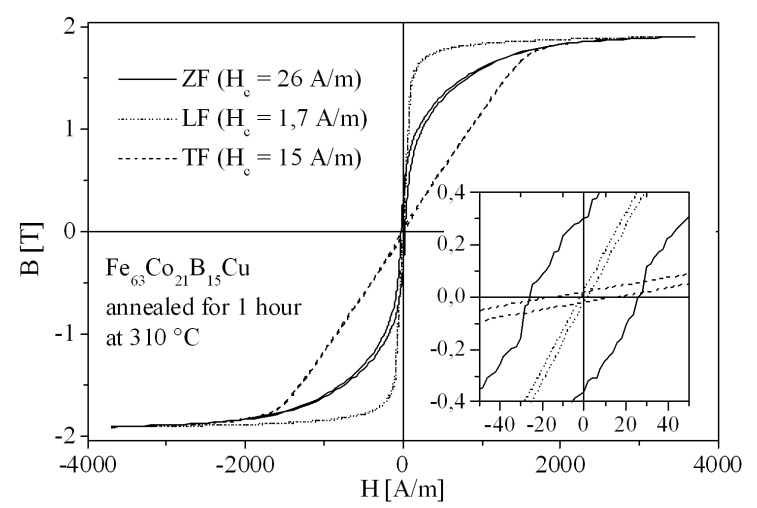

Fig. 1. Hysteresis loops for $\mathrm{Fe}_{63} \mathrm{Co}_{21} \mathrm{~B}_{15} \mathrm{Cu}$ alloy after different field annealing for $1 \mathrm{~h}$ at $310^{\circ} \mathrm{C}$.

after annealing in transverse magnetic field. A heat treatment under the presence of longitudinal magnetic field results in squared hysteresis loop that is characterized by a significant reduction of coercivity. The coercive field value for such thermally relaxed amorphous sample is $1.7 \mathrm{~A} / \mathrm{m}$ and the magnetic flux density after such heat treatment reaches $1.83 \mathrm{~T}$. These magnetic characteristics are improved in comparison with those obtained after longitudinal field annealing for $1 \mathrm{~h}$ at $320^{\circ} \mathrm{C}$, which induced a minute degree of the crystallization in the heat treated amorphous material [3].

In order to check the performance of a new generation of sensors based on the magnetic cores using such LF-annealed material, we have focused our attention to the improvement of the electronics of the relaxation sensors as well as to the modeling of sensor variables and characteristics. Real characteristics obtained for new sensor, together with results obtained by the simulation of its model in Matlab-Simulink environment are shown in Figs. 2 and 3. Figure 2 shows the measured and modeled conversion characteristics of sensor for positive excitation pulses $50 \mathrm{~mA}$, frequency $5 \mathrm{kHz}$ and the ratio of excitation to relaxation time equal to $2 / 5$. The model demonstrates a remarkable coincidence of the results of

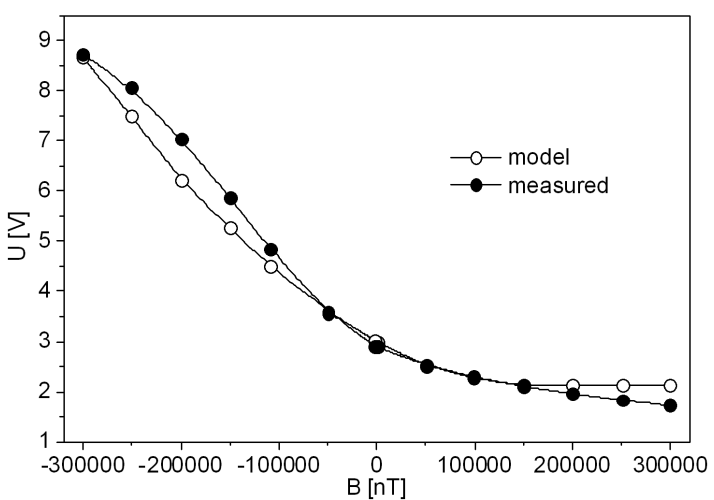

Fig. 2. Measured and modeled conversion characteristics of relaxation sensor for positive excitation pulses.

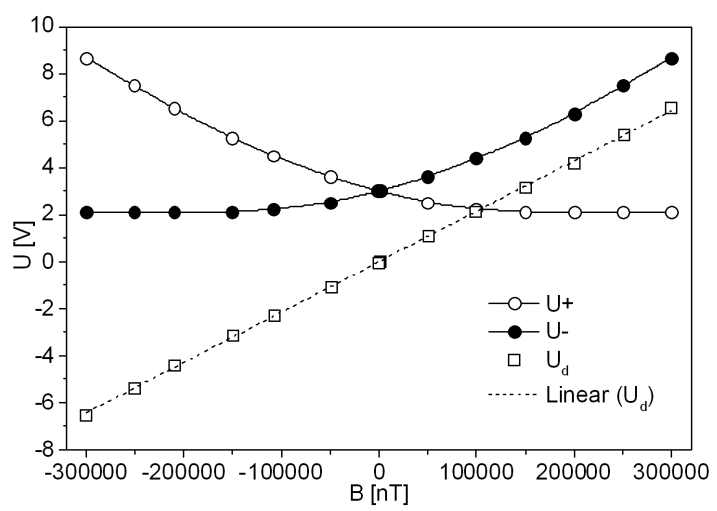

Fig. 3. Resulting conversion characteristic of relaxation sensor as differential voltage $U_{\mathrm{d}}$ of responses to positive $U+$ and negative $U-$ excitation.

simulations with those of the real experimental measurements. In the $100 \mu \mathrm{T}$ range, the deviation between the measured and modeled characteristics is smaller than $6 \%$ and the maximum deviation in the entire field range of $\pm 300 \mu \mathrm{T}$ is below $11.5 \%$.

The resulting conversion characteristic obtained as differential voltage between response of sensor to positive and negative excitation pulses is plotted in Fig. 3. This figure shows that a good linearity of $U_{\mathrm{d}}(B)$ is attained in the entire measuring range of $300 \mu \mathrm{T}$ and the sensitivity of the sensor in this operational field range is $2 \mathrm{~V} / 100 \mu \mathrm{T}$. The similar sensitivity is obtained also for the commercial VAC 25X material, which is currently used for this kind of sensors, however, the typical operational field range for VAC $25 \mathrm{X}$ is only $100 \mu \mathrm{T}$. The main advantage for the use of the melt-spun $\mathrm{Fe}_{63} \mathrm{Co}_{21} \mathrm{~B}_{15} \mathrm{Cu}$ alloys as core materials in the relaxation magnetic sensors is the extension of their linear measuring range as compared to the currently used commercial materials.

\section{Conclusion}

In summary we have demonstrated that the magnetic $B H$ characteristics of the field annealed melt-spun 
$\mathrm{Fe}_{63} \mathrm{Co}_{21} \mathrm{~B}_{15} \mathrm{Cu}$ alloy show good prospects for their use as a core material in the magnetic relaxation sensors. The saturation magnetic flux density for the longitudinal field annealed alloy reaches $1.83 \mathrm{~T}$ and the value of coercive field is $1.7 \mathrm{~A} / \mathrm{m}$. The high magnetic flux density markedly increase the linear measuring range of sensor while keeping the similar sensitivity as commercial amorphous VAC $25 \mathrm{X}$ materials currently used in these sensor cores.

\section{Acknowledgments}

This work has been supported by the Agency for the Research and Development SK under project APVV-
-0454-07. It was partially supported also by the projects VEGA 2/0209/10 and GDRE GAMAS.

\section{References}

[1] D. Praslička, IEEE Trans. Magn. 30, 934 (1994).

[2] J. Blažek, J. Hudák, D. Praslička, I. Mikita, Sensors Actuators, A Phys. 59, 123 (1997).

[3] I. Marcin, A. Klinda, P. Švec, D. Praslička, J. Blažek, J. Kováč, P. Švec Sr, I. Škorvánek, IEEE Trans. Magn. 46, 416 (2010). 